\title{
A new physiological role for CcpA in adaptation of Bacillus Subtilis to sugar-induced osmotic stress
}

\begin{abstract}
The model Gram-positive bacterium Bacillus subtilis is liable to be exposed to highsalinity environments in its natural habitats and is often used in fermentation with high concentrations of glucose or other sugars. High salinity or high concentrations of sugars can cause osmotic stress to $B$. subtilis. Past researches regarding osmoadaptation of $B$. subtilis were mainly focused on responses to salt-induced osmotic stress. There was little or no mention about how B. subtilis cells responded to sugar-induced osmotic stress. The catabolite control protein $(\mathrm{CcpA})$ is known to be a global transcriptional regulator that mediates glucose repression of many catabolic genes and activation of genes involved in excretion of excess carbon in various Bacillus species. However, the physiological significance for CcpA-mediated sugar activation of $\operatorname{deg} \mathrm{U}$, gltAB, opuA, opuE, proHJ and the ilvB operon remained poorly defined. Here based on the results from the literature search, it is now proposed that CcpA-mediated sugar activation of these osmoadaptive genes may facilitate the adaptation of $B$. subtilis to sugar-induced osmotic stress. This finding could add a new physiological role to CcpA in B. subtilis and probably its close relatives. As to biotechnological application, construction of $B$. subtilis strains that could over-express CcpA might potentially enhance their abilities to withstand higher concentrations of sugars for producing higher yields of various economically effective fermentation products.
\end{abstract}

Keywords: Bacillus subtilis, catabolite control protein, ccpa, osmoadaptation, sugar induced osmotic stress
Volume 5 Issue 4 - 2017

\section{Gwo Chyuan Shaw}

Institute of Biochemistry and Molecular Biology, National Yang Ming University, Taiwan

Correspondence: Gwo Chyuan Shaw, Institute of Biochemistry and Molecular Biology, School of Life Sciences, National Yang Ming University, Taipei I I 2, Taiwan, Tel 886-2 28267I27, Fax 886-2-28264843, Email gcshaw@ym.edu.tw

Received: June 01, 2017 | Published: July 31, 2017
Abbreviations: CcpA, catabolite control protein; PTS, phosphor transferase system

\section{Introduction}

The model Gram-positive bacterium Bacillus subtilis is liable to be exposed to high-salinity environments in its natural habitats and is often used in fermentation with high concentrations of glucose or other sugars. High salinity or high concentrations of sugars can cause osmotic stress to $B$. subtilis. ${ }^{1}$ The salt-induced osmotic stress can activate signalling pathways to induce expression of genes for biosynthesis or uptake of osmotically compatible solutes, thus protecting B. subtilis cells against the salt stress. Glycine betaine and proline are two important compatible solutes that can be utilized by $B$. subtilis to cope with salt stress. ${ }^{2}$ The salt-inducible opuA operon encodes an $\mathrm{ABC}$ transporter that is involved in the uptake of glycine betaine for defense of $B$. subtilis against salt stress. ${ }^{3} \mathrm{OpuE}$ is a proline uptake transporter that can be induced by high salinity for osmotolerance. ${ }^{4}$ The salt-inducible proHJ operon encodes enzymes involved in biosynthesis of proline for osmotic adaptation. 1 The two-component signal transduction system DegSU can sense high salinity and be induced by salt stress. The response regulator DegU is a positive regulator of the osmotic response. Mutation of $\operatorname{deg} U$ confers an osmosensitive phenotype to B. subtilis. ${ }^{5,6}$ Past researches regarding osmoprotection of $B$. subtilis were mainly focused on responses to salt-induced osmotic stress. There was little or no mention about how $B$. subtilis cells responded to sugar-induced osmotic stress. The catabolite control protein $(\mathrm{CcpA})$ is known to be a global transcriptional regulator that mediates glucose repression of many catabolic genes and activation of genes involved in excretion of excess carbon in various Bacillus species. ${ }^{7}$ A literature search has revealed that glucose can also CcpA-dependently activate expression of the $\operatorname{deg} \mathrm{U}$ gene, the glt $\mathrm{AB}$ operon, the ilvB operon, the opuA operon and the opuE gene..$^{8-11}$ The ilvB operon of $B$. subtilis encodes enzymes involved in biosynthesis of the branched-chain amino acid isoleucine. Salt-induced accumulation of isoleucine is known to play an important role in tolerance of plants to salt stress. ${ }^{12}$ The gltAB operon of $B$. subtilis encodes glutamate synthase. Glutamate is a precursor of proline biosynthesis and it per se can function as an osmotically compatible solute. ${ }^{13}$ A previous report has shown that sucrose and lactose can induce proHJ expression. ${ }^{1}$ Glucose can also activate proHJ expression via CcpA (CJ Lin and GC Shaw, unpublished observations). The biological significance for CcpA-mediated glucose activation of the glt $\mathrm{AB}$ operon or the ilvB operon was previously suggested to be a link between carbon and nitrogen metabolism. ${ }^{8,14}$ The physiological significance for CcpA-mediated glucose activation of $\operatorname{deg} U$ was postulated to be relevant with consumption of acetylcoenzyme A during polyketide synthesi. ${ }^{11}$. However, the biological significance for CcpA-mediated sugar activation of $\operatorname{deg} U$, gltAB, opuA, opuE, proHJ and the ilvB operon remained poorly defined. In Escherichia coli, ProP is a member transporter for uptake of proline and other osmoprotectants. ProP is also involved in sensing the osmotic stress caused by high salinity. ${ }^{15}$ In $B$. subtilis, the sensor for perception of the osmotic stress caused by high salinity or high concentrations of sugars has not yet been identified. Nevertheless, it is known that glucose is transported into B. subtilis cells by the glucose-specific phosphoenolpyruvate: sugar phosphotransferase system (PTS) encoded by the ptsGHI operon.16 EIICBA is the gene product of ptsG and is a membrane transporter responsible for glucose transport and phosphorylation. Expression of the ptsGHI operon is known to be glucose-inducible. ${ }^{16}$

\section{Conclusion}

Here based on the results from the literature search, it is now proposed that sugar (including glucose and probably fructose, sucrose 
or lactose) activation of these osmoadaptive genes via CcpA may facilitate the adaptation of $B$. subtilis to sugar-induced osmotic stress. EIICBA may possibly be involved in sensing the osmotic stress caused by glucose and transducing the signal to CcpA via HPr and/or $\mathrm{Crh}^{17}$ to activate osmoadaptive genes for adaptation of $B$. subtilis to glucose-induced osmotic stress (Figure 1). This finding could add a new physiological role to CcpA in B. subtilis and probably its close relatives. As to biotechnological application, construction of B. subtilis strains that could over-express CcpA might potentially enhance their abilities to withstand higher concentrations of sugars for producing higher yields of various economically effective fermentation products.

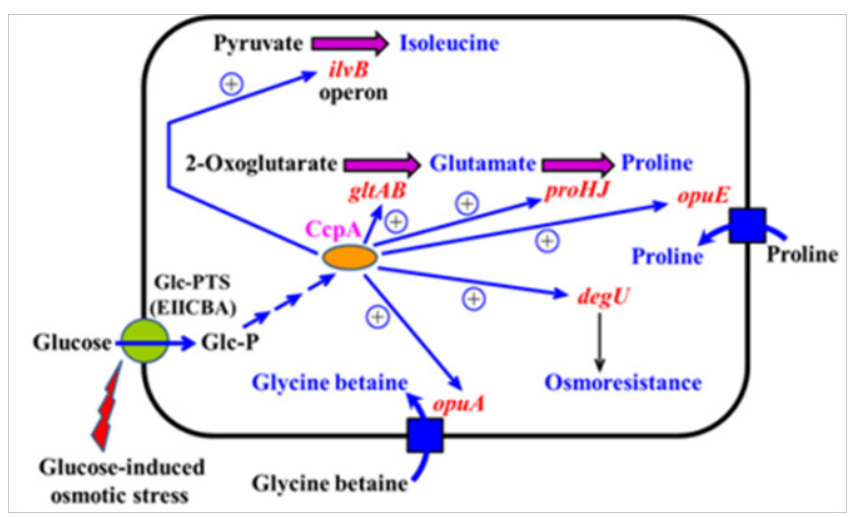

Figure I A model for the regulatory network of CcpA-mediated glucose activation of osmoadaptive genes for adaptation of $B$. subtilis to glucoseinduced osmotic stress. Osmoadaptive genes encode proteins responsible for biosynthesis or uptake of osmoprotectants such as glutamate, glycine betaine, isoleucine and proline. CсpA-mediated glucose activation of degU expression can confer protection of $B$. subtilis against glucose-induced osmotic stress. The glucose-inducible membrane transporter EIICBA may play a role in sensing the osmotic stress caused by high concentrations of glucose and transducing the signal to CсPA via HPr and/or Crh for activation of osmoadaptive genes.

Abbreviations: Glc-P, phosphorylated glucose; Glc-PTS, glucose-specific phosphoenolpyruvate: sugar phosphotransferase system

\section{Acknowledgements}

This work was supported by grant MOST 104-2311-B-010-006MY2 from the Ministry of Science and Technology of the Republic of China (Taiwan).

\section{Conflicts of interest}

No conflict of interest was declared.

\section{References}

1. Brill J, Hoffmann T, Bleisteiner M, Bremer E (2011) Osmotically controlled synthesis of the compatible solute proline is critical for cellular defense of Bacillus subtilis against high osmolarity. J Bacteriol 193(19): 5335-5346.

2. Kempf B, Bremer E (1998) Uptake and synthesis of compatible solutes as microbial stress responses to high-osmolality environments. Arch Microbiol 170(5): 319-330.
3. Kempf B, Bremer E (1995) OpuA, an osmotically regulated binding protein-dependent transport system for the osmoprotectant glycine betaine in Bacillus subtilis. J Biol Chem 270(28): 16701-16713.

4. von Blohn C, Kempf B, Kappes RM, Bremer E (1997) Osmostress response in Bacillus subtilis: characterization of a proline uptake system (OpuE) regulated by high osmolarity and the alternative transcription factor sigma B. Mol Microbiol 25(1): 175-187.

5. Ruzal SM, Sanchez-Rivas C (1998) In Bacillus subtilis DegU-P is a positive regulator of the osmotic response. Curr Microbiol 37(6): 368372

6. Steil L, Hoffmann T, Budde I, Volker U, Bremer E (2003) Genome-wide transcriptional profiling analysis of adaptation of Bacillus subtilis to high salinity. J Bacteriol 185(21): 6358-6370.

7. Fujita Y (2009) Carbon catabolite control of the metabolic network in Bacillus subtilis. Biosci Biotechnol Biochem 73(2): 245-259.

8. Wacker I, Ludwig H, Reif I, Blencke HM, Detsch C, et al. (2003) The regulatory link between carbon and nitrogen metabolism in Bacillus subtilis: regulation of the glt $\mathrm{AB}$ operon by the catabolite control protein CcpA. Microbiology 149(10): 3001-3009.

9. Lulko AT, Buist G, Kok J, Kuipers OP (2007) Transcriptome analysis of temporal regulation of carbon metabolism by CcpA in Bacillus subtilis reveals additional target genes. J Mol Microbiol Biotechnol 12: 82-95.

10. Marciniak BC, Pabijaniak M, de Jong A, Duhring R, Seidel G, et al. (2012) High- and low-affinity cre boxes for CcpA binding in Bacillus subtilis revealed by genome-wide analysis. BMC genomics 13: 401

11. Ishii H, Tanaka T, Ogura M (2013) The Bacillus subtilis response regulator gene degU is positively regulated by $\mathrm{CcpA}$ and by cataboliterepressed synthesis of ClpC. J Bacteriol 195(2): 193-201.

12. Joshi V, Joung JG, Fei Z, Jander G (2010) Interdependence of threonine, methionine and isoleucine metabolism in plants: accumulation and transcriptional regulation under abiotic stress. Amino acids 39(4): 933947

13. Wood JM (2011) Osmotic stress. In: Storz G, Hengge R (Eds.), Bacterial stress responses (2nd edn), ASM Press, Washington DC, USA, pp. 133156

14. Ludwig H, Meinken C, Matin A, Stulke J (2002) Insufficient expression of the ilv-leu operon encoding enzymes of branched-chain amino acid biosynthesis limits growth of a Bacillus subtilis ccpA mutant. J Bacteriol 184(18): 5174-5178

15. Keates RA, Culham DE, Vernikovska YI, Zuiani AJ, Boggs JM, et al. (2010) Transmembrane helix I and periplasmic loop 1 of Escherichia coli ProP are involved in osmosensing and osmoprotectant transport. Biochemistry 49(41): 8847-8856.

16. Stulke J, Martin Verstraete I, Zagorec M, Rose M, Klier A, et al. (1997) Induction of the Bacillus subtilis ptsGHI operon by glucose is controlled by a novel antiterminator, GlcT. Mol Microbiol 25(1): 65-78.

17. Galinier A, Haiech J, Kilhoffer MC, Jaquinod M, Stülke J, et al. (1997) The Bacillus subtilis crh gene encodes a HPr-like protein involved in carbon catabolite repression. Proc Natl Acad Sci USA 94(16): 84398444 . 